\title{
Three-dimensional non-linear instability of spontaneous fast magnetic reconnection
}

\author{
T. Shimizu, K. Kondoh, and M. Ugai \\ Department of Computer Science, Ehime University, Bunkyo town, Matsuyama City 790-8577, Japan
}

(Received November 2, 2007; Revised June 23, 2008; Accepted August 24, 2008; Online published May 29, 2009)

\begin{abstract}
Three-dimensional instability of spontaneous fast magnetic reconnection is studied using MHD (magnetohydro-dynamic) simulation. Previous two-dimensional MHD studies have demonstrated that, if a current-driven anomalous resistivity is assumed, two-dimensional fast magnetic reconnection occurs and two-dimensional largescale magnetic loops, i.e., plasmoids, are ejected from the reconnection region. In most two-dimensional MHD studies, the structure of the current sheet is initially one-dimensinal. On the other hand, in recent space plasma observations, fully three-dimensional magnetic loops frequently appear even in the almost one-dimensional current sheet. This suggests that the classical two-dimensional fast magnetic reconnection may be unstable to any three-dimensional perturbation, resulting in three-dimensional fast magnetic reconnection. In this paper, we show that a three-dimensional resistive perturbation destabilizes two-dimensional fast magnetic reconnection and results in three-dimensional fast magnetic reconnection. The resulting three-dimensional fast reconnection repeatedly ejects three-dimensional magnetic loops downstream. The obtained numerical results are similar to the pulsating downflows observed in solar flares. According to the Fourier analysis of the ejected magnetic loops, the time evolution of this three-dimensional instability is fully non-linear.
\end{abstract}

Key words: Magnetic reconnection, solar flare, MHD simulation.

\section{Introduction}

Fast magnetic reconnection provides a physical mechanism by which magnetic energy is explosively converted into plasma kinetic and thermal energies. This mechanism has been considered to play a crucial role in solar flares and geomagnetic substorms. Fast magnetic reconnection has been reported in many numerical magneto-hydro-dynamic (MHD) studies (e.g., Ugai and Tsuda, 1977). On the other hand, the plasma jets and magnetic loops associated with fast magnetic reconnection have been also reported in space satellite observations of solar flares (Masuda et al., 1994; Shibata et al., 1995; Shibata, 1996; Yokoyama et al., 2001; Asai et al., 2004). In particular, in some observation reports, the three-dimensional (3D) structure of fast magnetic reconnection was discussed (Shibata et al., 1995; Asai et al., 2004). Asai et al. (2004) reported that the intermittent plasma downflows observed in solar flares may be caused by fast magnetic reconnection in the high-altitude corona. In order to theoretically explain the observation data, they suggested that $3 \mathrm{D}$ fast magnetic reconnection may occur even in a uniform (1D) current sheet and, hence, the ejected 3D magnetic loops may be strongly localized in the current sheet direction. To explain the formation of impulsive plasma downflows, Linton and Longcope (2006) proposed a theoretical 3D model. However, it is still unclear how such a $3 \mathrm{D}$ fast magnetic reconnection process occurs in the $1 \mathrm{D}$ current sheet.

Copyright (c) The Society of Geomagnetism and Earth, Planetary and Space Sciences (SGEPSS); The Seismological Society of Japan; The Volcanological Society of Japan; The Geodetic Society of Japan; The Japanese Society for Planetary Sciences; TERRAPUB.
In this paper, the $2 \mathrm{D}$ spontaneous fast magnetic reconnection model proposed by Ugai is numerically developed to a 3D model. At this point, note that the study shown in this paper is basically different from the 3D studies reported by Ugai and collegues (Ugai et al., 2004, 2005). In the studies reported by Ugai, since the initial resistive disturbance is strongly localized in the sheet current direction, it directly results in a "single" 3D fast magnetic reconnection. In contrast, in the work presented here, the initial resistive disturbance is almost $2 \mathrm{D}$ but only includes a "very weak non-uniformity (perturbation)" in the sheet current direction, resulting in "multiple" $3 \mathrm{D}$ fast magnetic reconnection processes. In other words, this paper shows that the 2D fast reconnection process is basically unstable for a 3D perturbation. As a result, the destabilized 2D fast magnetic reconnection can be three-dimensionally localized even in a $1 \mathrm{D}$ current sheet. At the time, some 3D magnetic loops are repeatedly ejected from the reconnection region. These features of 3D fast reconnection seem to be consistent with the observation data of intermittent plasma downflows observed in solar flares.

\section{Simulation Setup}

In the spontaneous fast magnetic reconnection model, the reconnection process is initiated by a small resistive disturbance in the current sheet (Ugai and Shimizu, 1996; Ugai, 1999). After the initial disturbance is removed, fast reconnection spontaneously develops by a current-driven anomalous resistivity enhanced by the nonlinear tearing instability. Hence, no external driven mechanism is required to keep the reconnection process going. 
The MHD simulation procedures are basically the same as in Ugai (1987), except for the initial resistive disturbance shown later, in this article i.e., Eq. (1). The 3D compressible MHD equations are used. The computational region is restricted to the first quadrant and set to be a rectangular box, $\left(0 \leq x \leq L_{x}, 0 \leq y \leq L_{y}, 0 \leq z \leq L_{z}\right)$, because of the conventional symmetry boundary conditions with respect to the $x z$-plane at $y=0, y z$-plane at $x=0$, and $x y$-planes at $z=0$ and $L_{z}$. On the other boundaries, free boundary conditions are assumed, where all the quantities are determined by the state of the inner region, so that the first derivatives of the quantities in the direction normal to the boundaries vanish (the normal component of $\mathbf{B}$ is determined by the solenoidal condition). Because of the symmetry boundary conditions for $x y$-planes at $z=0$ and $L_{z}$, the simulation box is closed in the $z$-direction. In other words, the simulation box may be considered to be periodic in the $z$-direction, where $2 L_{z}$ is the maximum wave length for all the $z$-directional MHD waves. The computational region $L_{x}=30, L_{y}=10$, and $L_{z}=20$ are taken with the mesh points $N_{x}=880, N_{y}=1000$, and $N_{z}=100$.

\section{Simulation Results}

\subsection{The initial stage}

The simulation starts with $\beta_{0}=0.15$ in the magnetic field region, which consists of $B_{x 0}=1.0$ and $P_{0}=0.075$. Initially, every magnetic field line is exactly set up in the $x$-direction. The current sheet is initially formed on the $x z$ plane and the current is flowing in the negative $z$-direction. In the initial current sheet, $B_{x}$ monotonously varies from $B_{x 0}$ at $y=+1$ to zero at $y=0$. Then, $B_{x}=B_{x 0}$ is set for $y>+1$. There is no initial plasma flow. The Alfven speed $V_{\mathrm{A}}$ is unity in the initial upstream magnetic field region based on $B_{x 0}=1.0$, and the Alfven transit time to pass the distance of $L_{x}=30$ is set as $T=30$ in the time scale.

The initial resistive disturbance shown below initiates a tearing instability in the current sheet during $0<T<4$, which is strongly localized around the $z$-axis.

$$
\eta_{0}=0.04 \exp \left(-\alpha\left(x^{2}+y^{2}\right)\right)\left\{1+0.01 \cos \left(\pi z / L_{z}\right)\right\}
$$

where $\alpha=0.01$. In this initial resistive disturbance, the magnetic Reynolds number is estimated to be 25 at the origin. Note that this resistive disturbance fluctuates only by $1 \%$ in the $z$-direction, which is the sheet current direction. Accordingly, the resulting reconnection process is expected to be nearly 2D. In fact, the magnetic loop ejected firstly from the reconnection region is almost $2 \mathrm{D}$, as shown later in Fig. 1.

After $T=4, \eta_{0}$ is removed and, instead, the currentdriven anomalous resistivity $\eta_{1}$ shown below is switched on, which starts to drive the fast magnetic reconnection after about $T=20$.

$$
\eta_{1}\left\{\begin{array}{lll}
=0.002\left(V_{\mathrm{d}}-V_{\mathrm{c}}\right) & \text { for } & V_{\mathrm{d}} \geq V_{\mathrm{c}} \\
=0.0 & \text { for } & V_{\mathrm{d}}<V_{\mathrm{c}}
\end{array}\right.
$$

where $V_{\mathrm{d}}=|J| / \rho$ is the ion-electron drift velocity and $V_{\mathrm{c}}=4.0$ is set. The current density $J$ is obtained from

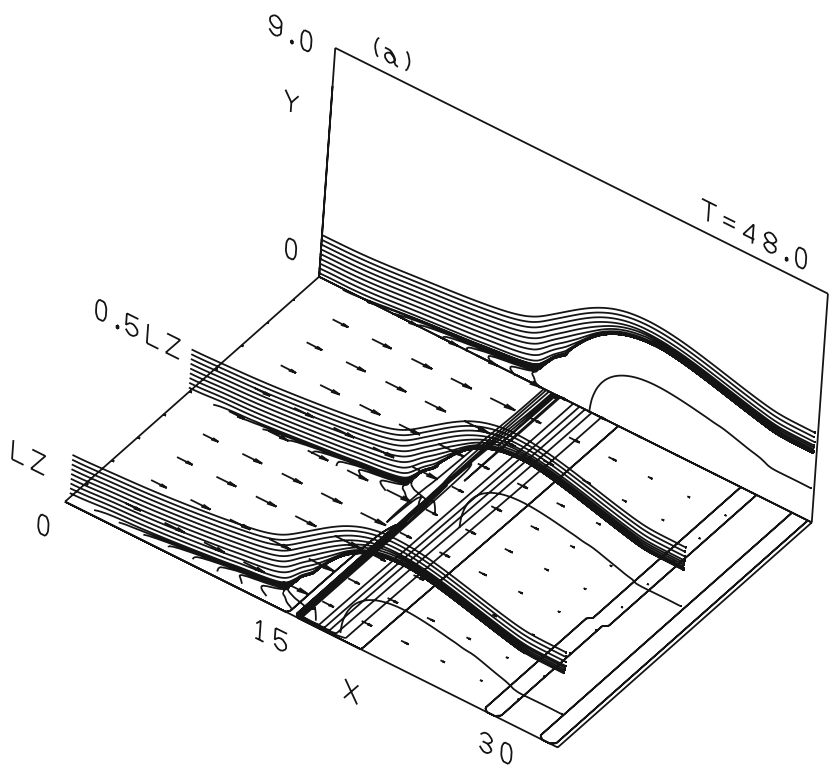

Fig. 1. Some of magnetic field lines, a contour map of $B_{y}$ field intensity, and a pattern of flow velocity at $T=48$.

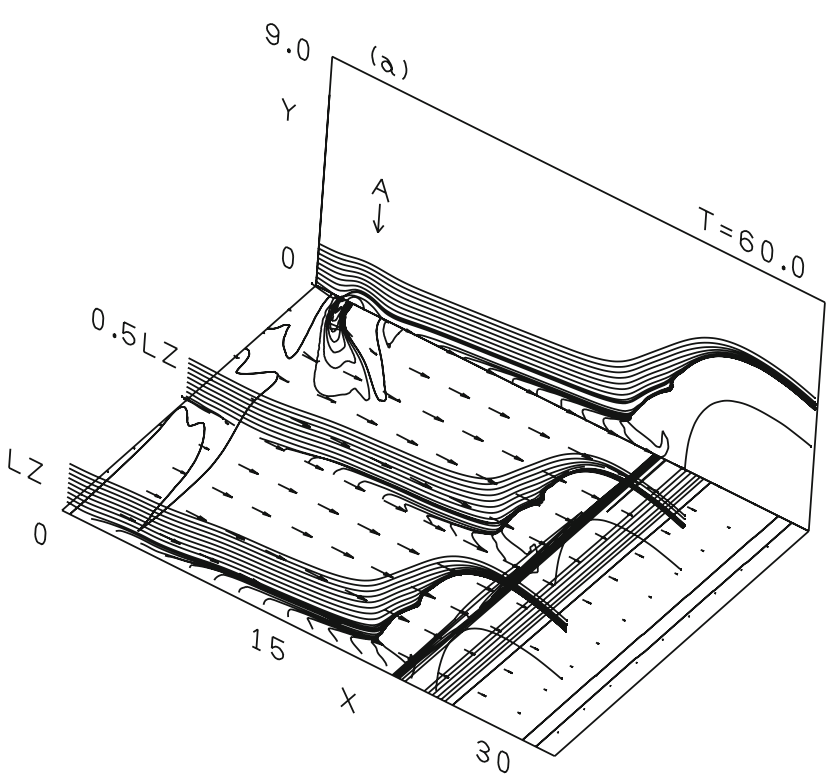

Fig. 2. The figure format is the same as Fig. 1 but at $T=60$.

rotB and is unity at the center of the initial current sheet, i.e., $y=0$. Also, the uniform plasma density $\rho$ is initially set to be unity.

\subsection{The first stage (2D fast reconnection)}

Figure 1 shows magnetic field lines on three $x y$-planes for $z=0,0.5 L_{z}$, and $L_{z}$ with a $B_{y}$ contour map and plasma flow vectors on an $x z$-plane for $y=0$ at $T=48$. Along the $z$-axis, the magnetic neutral line for fast reconnection process is formed. The magnetic field lines in all three $x y$ planes are very similar. In addition, the $B_{y}$ contour map and plasma flow vectors are almost uniform in the $z$-direction. Hence, at this time, the fast magnetic reconnection process is $2 \mathrm{D}$, and a $2 \mathrm{D}$ large-scale magnetic loop is formed between $15<x<30$. 


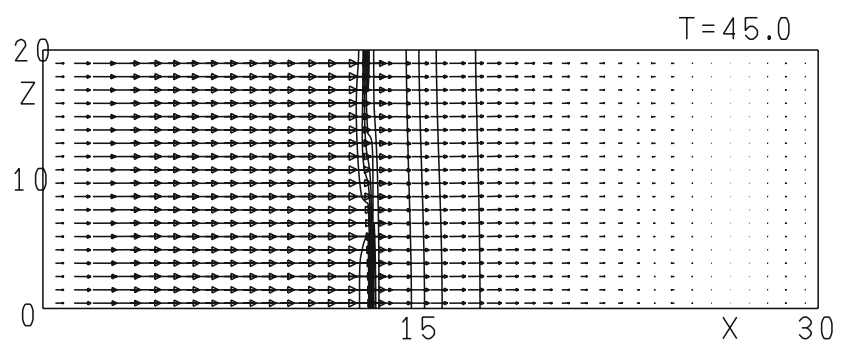

Fig. 3. $B_{y}$ contour map on the $x z$-plane at $y=0$ and $T=45$.

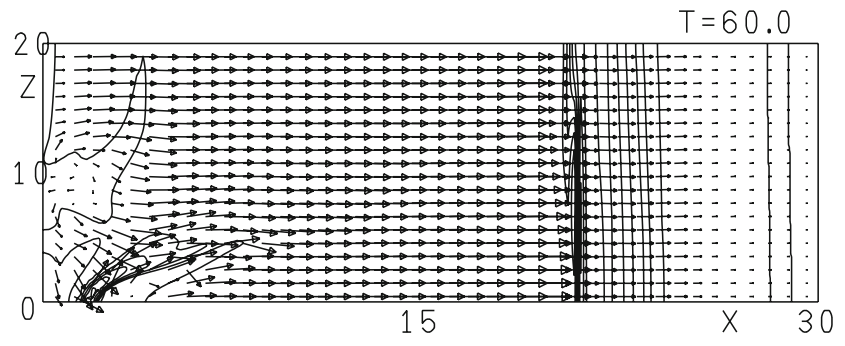

Fig. 4. $B_{y}$ contour map at $T=60$.

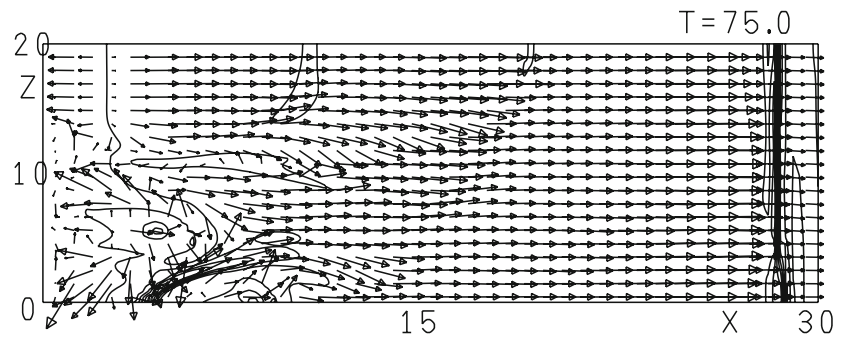

Fig. 5. $B_{y}$ contour map at $T=75$.

\subsection{The second stage (3D fast reconnection)}

Figure 2 shows the numerical result similar to Fig. 1 but for $T=60$. The 2D large-scale magnetic loop in $20<x<30$ corresponds to the one in $15<x<30$ of Fig. 1, so the loop is propagating with the reconnection jets. More exactly, the propagating speed is about $0.6 V_{\mathrm{A}}$ and is a little slower than the jet speed $V_{\mathrm{A}}$. In addition, another small magnetic loop is observed in the vicinity of the origin, which is marked by "A" in Fig. 2. Since this magnetic loop is not observed in the magnetic field lines of $z=0.5 L_{z}$ and $L_{z}$, the loop structure is evidently $3 \mathrm{D}$. This $3 \mathrm{D}$ magnetic loop is a result of the $z$-directional perturbation in the initial resistive disturbance Eq. (1). In Fig. 2, the $B_{y}$ contour map on the $x z$-plane at $y=0$ is also shown, which is the intensity of the reconnected magnetic field. Clearly, the 3D magnetic loop marked by "A" corresponds to a highintensity island on the $B_{y}$ contour map, which is a little extended in the $z$-direction and largely curved in the positive $x$-direction.

\subsection{Time variation of $B_{y}$ contour map}

Figures 3, 4, and 5 show $B_{y}$ contour maps on the $x z$-plane for $y=0$ at $T=45,60$, and 75. In Fig. 3, no 3D magnetic loop is observed and a 2D magnetic loop is only observed as vertical bands at $13<x<17$. This is consistent with Fig. 1. Figure 4 is exactly the same as the contour map in Fig. 2, where $L_{z}=20$. In Fig. 4, a 3D magnetic

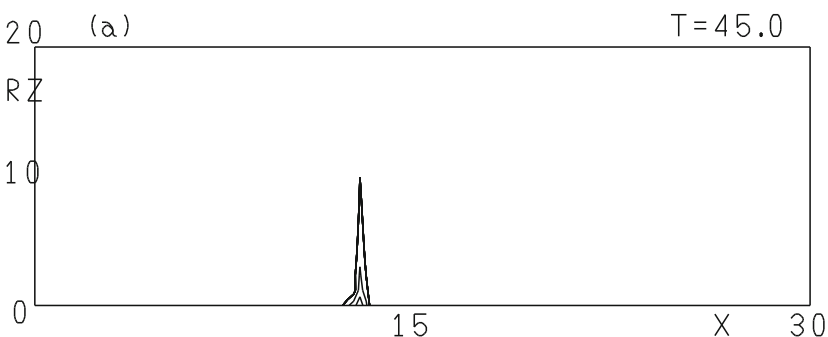

Fig. 6. Z-directional Fourier analysis of $B_{y}$ contour map on $x z$-plane at $T=45$.

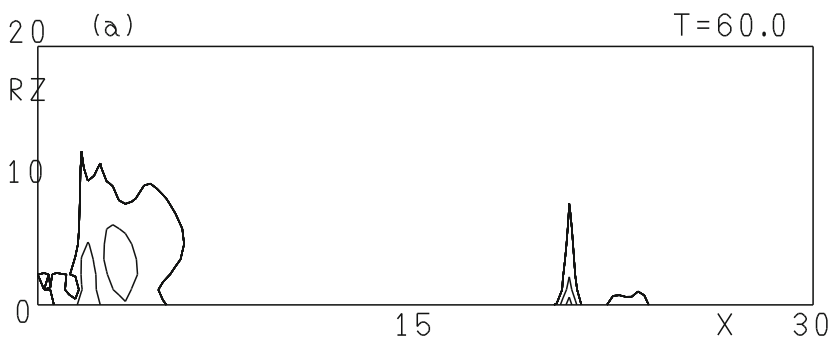

Fig. 7. Z-directional Fourier analysis at $T=60$.

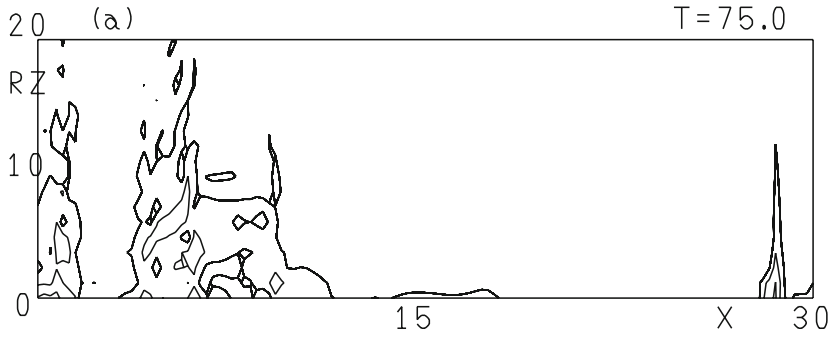

Fig. 8. Z-directional Fourier analysis at $T=75$.

loop appears and then, in Fig. 5, slightly propagates in the positive $x$-direction. In addition, in Fig. 5, considering the symmetry boundary condition on the $x$-axis, i.e., $z=0$, two 3D magnetic loops are also observed as small round-shape islands at $(x, z)=(5,5)$ and $(7,0)$.

\section{$3.5 z$-directional Fourier analysis}

Figures 6, 7, and 8 respectively show the $z$-directional Fourier analysis of Figs. 3, 4, and 5 as the contour map of the Fourier components. These figures show the time variation from $T=45$ to 75 . The vertical axis $R_{z}$ of these figures is scaled as the inverse of the $z$-directional wave length $\lambda_{z}$, where unity $R_{z}=\left(1 / \lambda_{z}\right)=1$ is equivalent to $1 /\left(2 L_{z}\right)$. Note that $2 L_{z}$ corresponds to the wave length of the $z$-directional resistive perturbation in Eq. (1). In Fig. 6, the growth of the Fourier components $1 \leq R_{z}<10$ related to the 2D magnetic loop is observed at $x=13$. In Fig. 7, the Fourier components move to $x=21$, and new Fourier components $1 \leq R_{z}<10$ widely appear in the 3D magnetic loop region located in $2<x<5$. Next, in Fig. 8, the new Fourier components widely spread in the $x$-direction, because of the multiple formations of 3D magnetic loops in Fig. 5. Since the initial resistive disturbance Eq. (1) only has $R_{z}=1$ component, the higher-order components $R_{z} \geq 2$ are generated by the non-linear instability of the fast magnetic reconnection. In Figs. 6 and 7, the 2D magnetic 


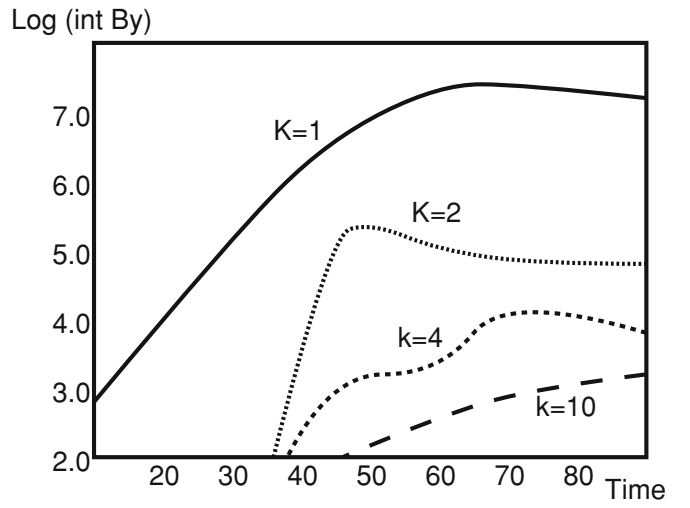

Fig. 9. Time variation of some $z$-directional Fourier components which are averaged over $0<x<L_{x}$

loop also generates the higher-order components $R_{z} \geq 2$. However, evidently, the higher-order Fourier components related to $3 \mathrm{D}$ magnetic loops are spread more widely than those of the 2D magnetic loop.

Figure 9 shows the time variation of four typical $z$ directional Fourier components for the $B_{y}$ contour map, where $k=R_{z}$. On the 2D stage $(T<50)$, the $k=1 \mathrm{com}$ ponent that corresponds to the $z$-directional perturbation in Eq. (1) linearly grows up and, on the 3D stage $(50<T)$, almost saturates. As mentioned in Figs. 7 and 8, since Eq. (1) has no $R_{z}=k \geq 2$ components, such higher-order components should be created from the $k=1$ component, due to the non-linear instability in the 3D fast magnetic reconnection. In fact, the higher-order components rapidly grow up after the linear growth of $k=1$.

\section{Summary}

In this paper, we have shown that 2D fast magnetic reconnection caused by a current-driven anomalous resistivity is unstable to a $3 \mathrm{D}$ resistive perturbation. In fact, in this MHD model, the initial current sheet has exactly a 1D structure, and every setup is exactly prepared for 2D fast magnetic reconnection, except for the initial 3D resistive perturbation $\eta_{0}$. At this point, note that the $3 \mathrm{D}$ fast reconnection process reported by Ugai (Ugai et al., 2004, 2005) is largely different because the initial resistive disturbance employed in his papers includes a largely non-uniform component along the neutral line and, hence, the resulting fast reconnection processes is fully 3D even on the first stage. In contrast, as shown in Fig. 1, the fast reconnection studied in this paper is still 2D on the first stage. The 3D fast reconnection is spontaneously caused after the $2 \mathrm{D}$ fast reconnection has been established. In addition, as shown in the time variation of Fig. 9, the $z$-directional higher-order Fourier components generated by the 3D instability rapidly grows after the $2 \mathrm{D}$ stage. This means that the 3D instability shown here is fully non-linear.

As shown in Fig. 5, the appearance of the multiple 3D magnetic loops means that $3 \mathrm{D}$ fast reconnection process is pulsating. This may support the intermittent downflow reported by Asai et al. (2004), where 3D magnetic loops may be repeatedly ejected even on the $1 \mathrm{D}$ current sheet, due to the fast magnetic reconnection.

In the next step of this study, the temporal dynamics of ejected magnetic loops must be studied. In the temporal dynamics, some of the ejected magnetic loops may merge into a magnetic loop in the downstream region. Finally, few large-scale magnetic loops can only survive. At this point, the 3D fast reconnection model shown in this paper may be closely related to the fractal and intermittent reconnection model that was proposed by Shibata and Tanuma (2001) to explain the solar flare observation data as a time-development from micro-reconnection processes to a macro-reconnection process. Since their basic concept is a $2 \mathrm{D}$ model, the $3 \mathrm{D}$ fast reconnection shown here should give a new view point to their model.

Acknowledgments. This work was supported by grant-in-aids of the Japanese education ministry; Ehime Univ. T. Shimizu, "Study of Multi-scale Plasma Simulation of Magnetic Reconnection" (15540475) and Kyoto Univ. K. Shibata, Creative Scientific Research, "The Basic Study of Space Weather Prediction" (17GS0208). In addition, this work is supported by Research Institute for Sustainable Humanosphere (RISH) of Kyoto University and Solar-Terrestrial Environment Laboratory (STEL) of Nagoya University. The numerical calculations were performed on the KDK computer system at RISH and FUJITSU HP2500 parallel computer system at Nagoya and Kyoto University Data Processing Centers.

\section{References}

Asai, A., T. Yokoyama, M. Shimojo, and K. Shibata, Downflow motions associated with Impulsive nonthermal emissions observed in the 2002 July 23 Solar Flare, Astrophys. J., 605, L77, 2004.

Linton, M. and D. Longcope, A Model for Patchy Reconnection in Three Dimensions, Astrophys. J., 642, 1177-1192, 2006.

Masuda, S., T. Kosugi, H. Hara, S. Tsuneta, and Y. Ogawara, Loop-top impulsive hard X-ray source of a solar flare as evidence for magnetic reconnection, Nature, 371, 495-497, 1994.

Shibata, K., New Observational Facts about Solar Flares from Yohkoh Studies-Evidence of Magnetic Reconnection and a Unified Model of Flares, Adv. Space Res., 17, 9-18, 1996.

Shibata, K. and S. Tanuma, Plasmoid-induced-reconnection and fractal reconnection, Earth Planets Space, 53, 473-482, 2001.

Shibata, K., S. Masuda, M. Shimojo, H. Hara, T. Yokoyama, S. Tsuneta, T. Kosugi, and Y. Ogawara, Hot plasma ejections associated with Compact-Loop solar flares, Astrophys. J., 451, L83, 1995.

Ugai, M., Strong loop heating by the fast reconnection in a closed system, Geophys. Res. Lett., 14, 103-106, 1987.

Ugai, M., Basic physical mechanism of reconnection development and magnetic loop dynamics, J. Geophys. Res., 104(A4), 6929-6939, 1999.

Ugai, M. and T. Tsuda, Magnetic field-line reconnexion by localized enhancement of resistivity, 1. Evolution in a compressible MHD fluid, $J$. Plasma Phys., 17, 337-351, 1977.

Ugai, M. and T. Shimizu, Computer studies on the spontaneous fast reconnection mechanism in three dimensions, Phys. Plasmas, 3, 853-862, 1996.

Ugai, M., K. Kondoh, and T. Shimizu, Computer studies on the threedimensional spontaneous fast reconnection model as a nonlinear instability, Phys. Plasmas, 11, 1416-1423, 2004.

Ugai, M., K. Kondoh, and T. Shimizu, Spontaneous fast reconnection in three dimensions, Phys. Plasmas, 12, 042903, 2005.

Yokoyama, Y., K. Akita, T. Morimoto, K. Inoue, and J. Newmark, Clear evidence of reconnection inflow of a solar flare, Astrophys. J., 546, L69, 2001 .

T. Shimizu (e-mail: shimizu@cosmos.ehime-u.ac.jp), K. Kondoh, and M. Ugai 